\title{
Modelling on c-Si/a-Si:H wire solar cells: some key parameters to optimize the photovoltaic performance
}

\author{
I. Ngo, M.E. Gueunier-Farret ${ }^{\mathrm{a}}$, J. Alvarez, and J.P. Kleider \\ LGEP, UMR 8507 CNRS, SUPELEC, UPMC, Université Paris-Sud 11, 11 rue Joliot-Curie, Plateau de Moulon, \\ 91192 Gif-sur-Yvette Cedex, France
}

Received: 21 November 2011 / Accepted: 23 April 2012

Published online: 18 July 2012

\begin{abstract}
Solar cells based on silicon nano- or micro-wires have attracted much attention as a promising path for low cost photovoltaic technology. The key point of this structure is the decoupling of the light absorption from the carriers collection. In order to predict and optimize the performance potential of $p$ - (or $n$-) doped c-Si/ $n$-(or $p$-) doped a-Si:H nanowire-based solar cells, we have used the Silvaco-Atlas software to model a single-wire device. In particular, we have noticed a drastic decrease of the open-circuit voltage $\left(V_{o c}\right)$ when increasing the doping density of the silicon core beyond an optimum value. We present here a detailed study of the parameters that can alter the $V_{o c}$ of $c-\mathrm{Si}(p) / \mathrm{a}-\mathrm{Si}: \mathrm{H}(n)$ wires according to the doping density in c-Si. A comparison with simulation results obtained on planar c-Si/a-Si:H heterojunctions shows that the drop in $V_{o c}$, linked to an increase of the dark current in both structures, is more pronounced for radial junctions due to geometric criteria. These numerical modelling results have lead to a better understanding of transport phenomena within the wire.
\end{abstract}

\section{Introduction}

Solar cells based on Si micro- or nanowire arrays with radial $p$ - $n$ junction are of great interest as potentially lowcost solutions in solar cell production and have been extensively studied in the last years [1-6]. Unlike planar structures, photon absorption and minority carrier collection are decoupled as shown in Figure 1, and are not anymore in competition when optimizing the cell dimensions. In fact, the wires can be grown long enough to optimize light absorption while being small enough in radius to collect photo-generated carriers. This last property enables the use of low purity Si substrates with a short minority carrier diffusion length.

The vapour-liquid-solid (VLS) technique [7] assisted by gold catalyst is one of the most current methods to grow micro- and nano-wires for photovoltaic applications. Despite many advantages in using Au as VLS catalyst (non toxicity, chemical stability, availability), Au is known to diffuse in the nanowires [8] creating deep-level defects in $\mathrm{Si}$ and leading to an increase of the carrier recombination rate. Among the large number of catalyst materials that have been tested to replace $\mathrm{Au}$ [9], aluminium is a good candidate since it does not create deeplevel defects. Nevertheless, $\mathrm{Al}$ is a $p$-type dopant and the wires can be strongly $p$-doped. It is not obvious to know whether the performances of the solar device are strongly influenced by such a $p$-type doping level. In that case,

${ }^{\text {a }}$ e-mail: farret@lgep.supelec.fr

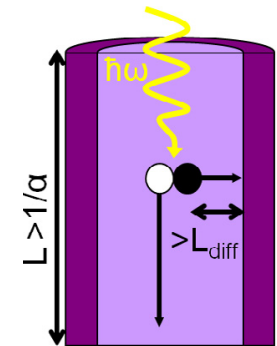

(a)

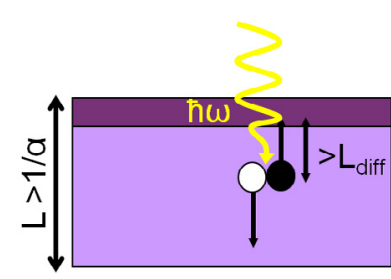

(b)
Fig. 1. Structures of solar cells based on (a) a radial $p$ - $n$ junction, (b) a planar $p$ - $n$ junction. Light penetration into the cell is characterized by the parameter $1 / \alpha, \alpha$ being the wavelengthdependent absorption coefficient. The diffusion length of the generated minority carriers is given by $L_{\text {diff }}$. In the case of the radial junction, light absorption and carrier collection are decoupled.

numerical modelling can be of great help. In this modelling work, we have studied one single-wire Si solar cell which consists in a $p$-type crystalline silicon (c-Si) core surrounded by a $n$-type hydrogenated amorphous silicon (a$\mathrm{Si}: \mathrm{H})$ thin layer. Cell efficiency, open-circuit voltage $\left(V_{o c}\right)$, short-circuit current $\left(I_{s c}\right)$ and fill factor $(\mathrm{FF})$ under AM 1.5 spectrum have been calculated as a function of the $p$ type doping density $\left(N_{a}\right)$ in the core. A drastic decrease in $V_{o c}$ has been observed when $N_{a}$ reaches values higher than $1 \times 10^{17} \mathrm{~cm}^{-3}$. An insight into the transport properties of the wire in the dark can help to understand this behavior. 


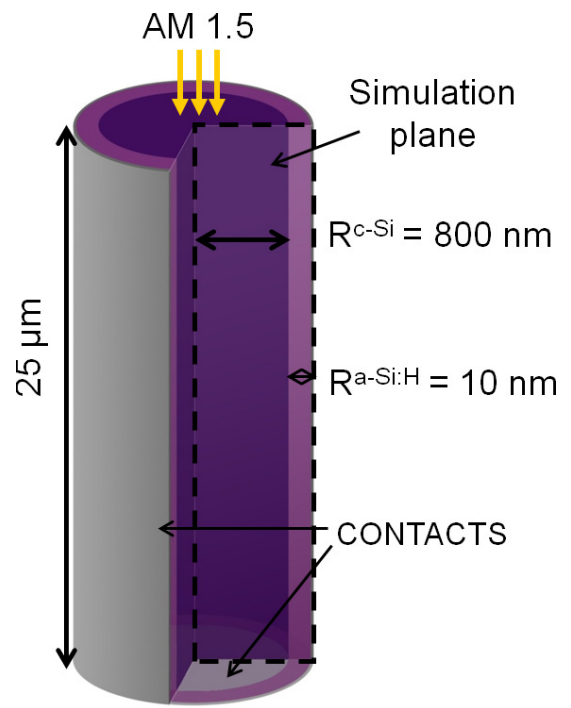

Fig. 2. Single-wire geometry of the model.

\section{Silicon nanowire modelling: geometry and physical parameters}

Simulations of the electrical characteristics of a singlewire c-Si/a-Si:H radial heterojunction solar cell were performed using ATLAS Silvaco software [10]. The geometry of the radial $p$ - $n$ junction studied here is given in Figure 2. The design was defined as a simulation plane in 2-dimensional cylindrical coordinates which models the $3 \mathrm{D}$ cylindrical structure. The wire dimensions were chosen according to experimental data of fabricated wire array cells, with a wire length of $25 \mu \mathrm{m}$ and a radius of $810 \mathrm{~nm}$. The $p$-type c-Si core (radius of $800 \mathrm{~nm}$ ) is surrounded by a thin layer $(10 \mathrm{~nm})$ of $n$-type doped a-Si:H. Ohmic contacts were used, one at the bottom of the structure on the crystalline base and another one around the wire on the amorphous layer. At the bottom contact, the electron surface recombination velocity has been taken equal to $50 \mathrm{cms}^{-1}$. For the surrounded contact, flat band conditions were imposed.

For the a-Si:H layer, the distribution of the density of states (DOS) is made of two exponential band tails (valence and conduction band tails) and two Gaussian distributions of deep defect states, one donor-like and one acceptor-like. The DOS and doping concentration were adjusted such that the Fermi level at room temperature was set at $0.2 \mathrm{eV}$ below the conduction band edge $E_{c}$. The electron and hole mobilities were taken equal to $\mu_{n}=20 \mathrm{~cm}^{2} \mathrm{~V}^{-1} \mathrm{~s}^{-1}$ and $\mu_{p}=5 \mathrm{~cm}^{2} \mathrm{~V}^{-1} \mathrm{~s}^{-1}$, respectively. In the c-Si core, we fixed a value of $1 \mathrm{~ms}$ for the carrier lifetime which corresponds to a diffusion length much bigger than the wire radius. The doping density $N_{a}$ was varied between $1 \times 10^{15} \mathrm{~cm}^{-3}$ and $1 \times 10^{19} \mathrm{~cm}^{-3}$ such that the Fermi level at room temperature moves from $0.25 \mathrm{eV}$ above the valence band edge $E_{v}$ to a position very close to $E_{v}$, respectively.

The simulation is based on the resolution of Poisson's equation and electron and hole continuity equations. The
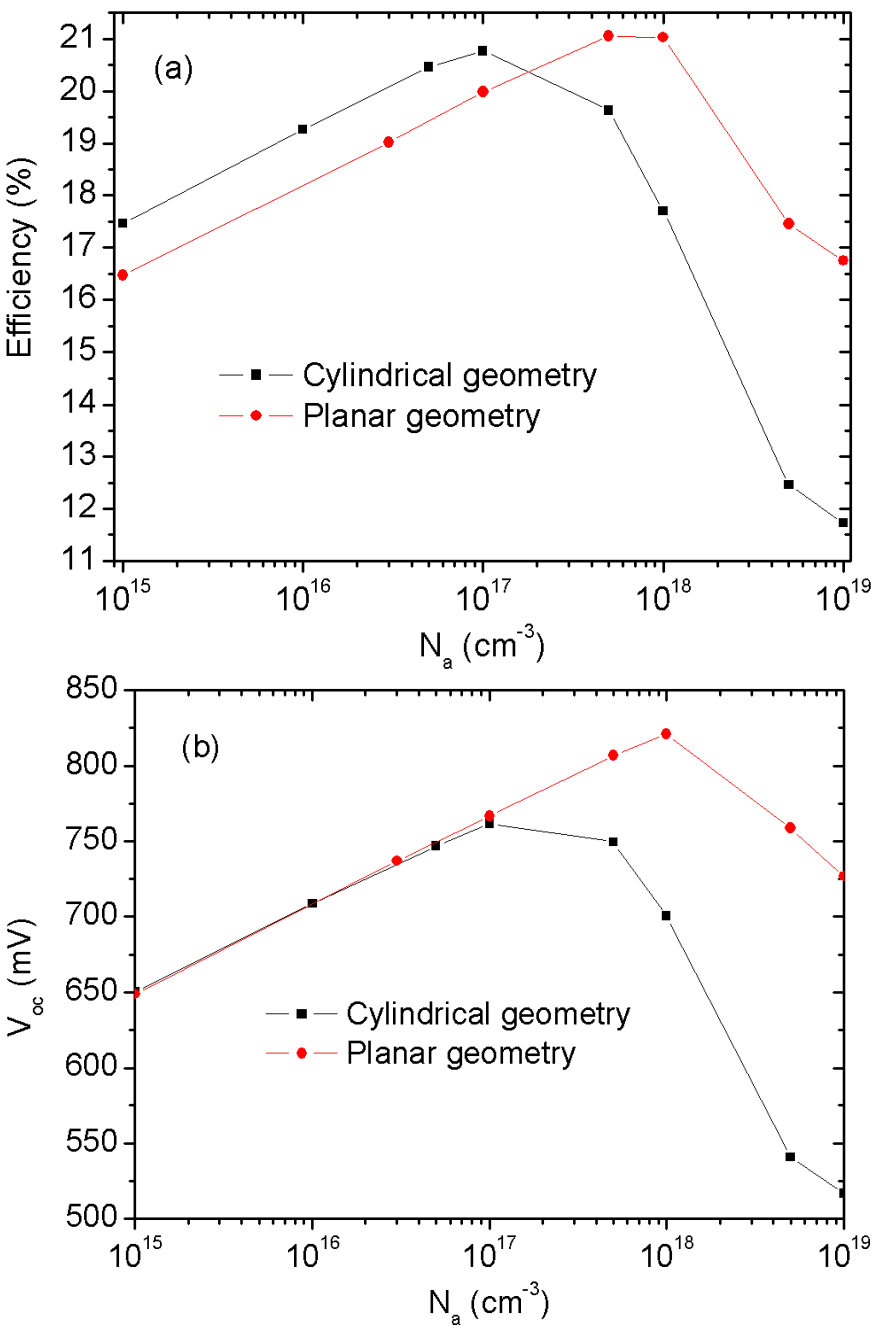

Fig. 3. Variations of the efficiency (a) and of the open-circuit voltage $V_{o c}(\mathrm{~b})$ of the modeled single-wire cell as a function of the $p$-type doping density $\mathrm{Na}$ in the c-Si core. These results are compared to those calculated for an equivalent c-Si $(p) / \mathrm{a}-\mathrm{Si}: \mathrm{H}(n)$ planar structure.

Boltzmann statistics is used with the drift-diffusion model in ATLAS, and Shockley-Read-Hall recombination was considered. The AM1.5 solar spectrum was used for the optical generation, the wire being under vertical illumination (see Fig. 2). Current-voltage characteristics $(I(V))$ were calculated in the dark and under standard onesun illumination conditions. The open-circuit voltage, the short-circuit current, and the efficiency of the cell were deduced from the $I(V)$ curves under illumination.

\section{Simulation results and discussion}

The variations of the efficiency and of the open-circuit voltage of the single-wire device is given as a function of the doping density $N_{a}$ in Figures $3 \mathrm{a}$ and $3 \mathrm{~b}$, respectively. These variations are compared to those of an equivalent planar solar cell. The planar structure was defined as a thin a-Si:H $(n)$ layer $(10 \mathrm{~nm})$ on a $25 \mu$ m-thick c-Si $(p)$ 


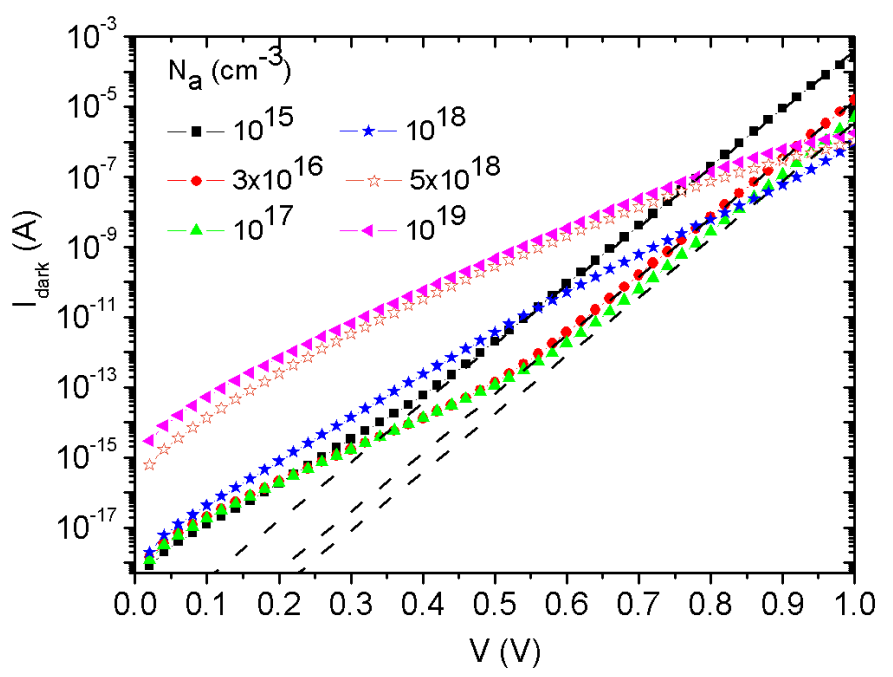

Fig. 4. Forward dark current characteristics $I_{\text {dark }}(V)$ for different doping density $N_{a}$ in the c-Si $(p)$ core of the wire. For $N_{a} \leqslant 1 \times 10^{17} \mathrm{~cm}^{-3}$, the fits of the curves with the exponential law $I_{0} \exp (q V / k T)$ corresponding to an ideal diode behavior is also given (dash lines).

wafer. The material properties and the illuminated surface are strictly the same in both structures. Moreover, whatever the doping density in the studied range, the shortcircuit current density was found constant and equal to $32.1 \mathrm{~mA} / \mathrm{cm}^{2}$ for both cylindrical and planar geometries.

It can be observed on Figures $3 \mathrm{a}$ and $3 \mathrm{~b}$ that the efficiency and $V_{o c}$ increase with $\log \left(N_{a}\right)$ when $N_{a}$ increases until an optimum value $N_{a o p t}$ which differs in both structures. In the case of the wire cell, $N_{\text {aopt }}$ is found equal to $1 \times 10^{17} \mathrm{~cm}^{-3}$. Beyond $N_{\text {aopt }}$, there is a strong decrease of the efficiency which is mainly due to a heavy drop in the $V_{o c}$, and this behavior is more important in the silicon wire than in the planar device.

The $V_{o c}$ is given by the relationship: $V_{o c}=n k T / q$ $\ln \left(I_{s c} / I_{o}\right)$, where $n$ is the diode ideality factor, $k$ the Boltzmann's constant, $T$ the temperature, $q$ the electronic charge and $I_{O}$ the dark reverse saturation current. Since $I_{s c}$ remains constant with $N_{a}$, the variation of the $V_{o c}$ is related to the dark current. Thus, for the single-wire structure, the $I(V)$ characteristics in the dark $\left(I_{\text {dark }}\right)$ were calculated for different values of $N_{a}$ between $1 \times 10^{15} \mathrm{~cm}^{-3}$ and $1 \times 10^{19} \mathrm{~cm}^{-3}$ and the results are presented in Figure 4.

At low doping concentration $\left(N_{a} \leqslant 1 \times 10^{17} \mathrm{~cm}^{-3}\right)$, the trend of the curves remains the same and $I_{d a r k}$ decreases when $N_{a}$ increases, which is a classical behavior in a $p$ - $n$ junction. A change in the slope of the curves can be observed, which indicates two current regimes: at high forward bias $(V \geqslant 0.5 \mathrm{~V})$, the $I_{\text {dark }}(V)$ curves can be fitted by the expression $I_{d a r k}=I_{0} \exp (q V / k T)$ as shown in Figure 4 , which corresponds to an ideal diode behavior with a diffusion current. The values of $I_{0}$ deduced from these fits are found inversely proportional to $N_{a}$, which explains the variations of the $V_{o c}$ previously described in this range of $N_{a}$. At low forward bias, the dark current seems to be dominated by a recombination/generation current in the

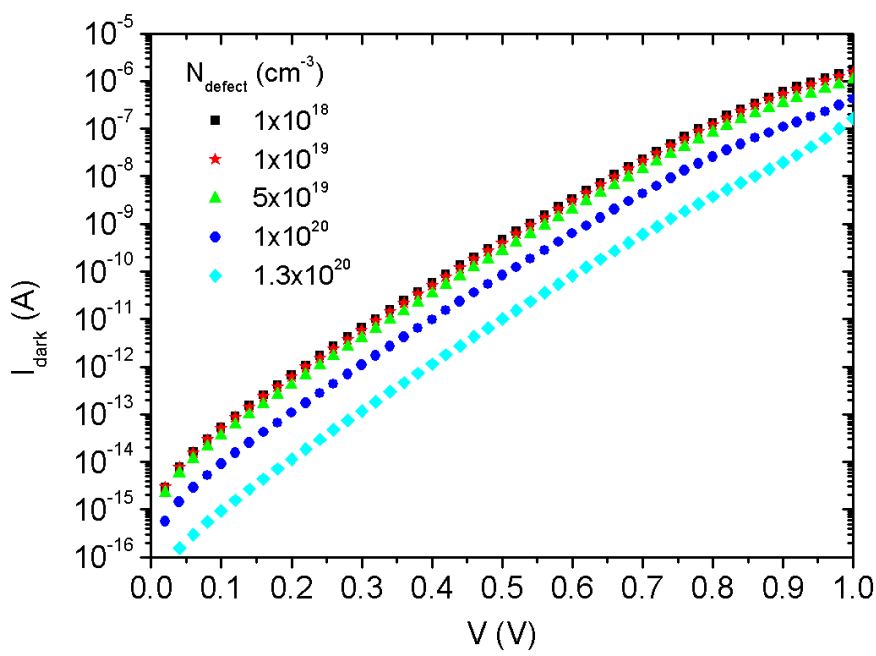

Fig. 5. Forward dark current characteristics $I_{\text {dark }}(V)$ for various values of the defect density $N_{\text {defect }}$ in the a-Si:H layer and a given doping density $N_{a}$ in the c-Si $(p)$ core equal to $1 \times 10^{19}$ $\mathrm{cm}^{-3}$.

a-Si:H layer. At high doping density $\left(N_{a}>1 \times 10^{17} \mathrm{~cm}^{-3}\right)$, no part of the $I_{\text {dark }}(V)$ characteristic at forward bias can be fitted by the previous exponential law and the recombination current in the a-Si:H shell becomes predominant all over the forward bias range. This is illustrated in Figure 5 where $I_{\text {dark }}(V)$ characteristics are calculated for various defect density $N_{\text {defect }}$ in the a-Si:H layer and a given value of $N_{a}$ equal to $1 \times 10^{19} \mathrm{~cm}^{-3}$. It can be observed that the dark current in the wire decreases when $N_{\text {defect }}$ increases.

The heavy drop in $V_{o c}$ at high doping density of the c-Si $(p)$ core is directly related to a strong increase of the dark current which, at such $N_{a}$ values, mainly becomes a recombination current in the defect-states rich a-Si:H layer. However, according to Figure 3b, this behavior is much more pronounced for the radial junction than for the planar one (it has to be reminded that this comparison is presented for the same illuminated area in both structures). A mapping of the dark current components near the $\mathrm{c}-\mathrm{Si}(p) / \mathrm{a}-\mathrm{Si}: \mathrm{H}(n)$ heterointerface of the single wire is presented in Figure 6 for $N_{a}=1 \times 10^{17} \mathrm{~cm}^{-3}$ and $N_{a}=1 \times 10^{19} \mathrm{~cm}^{-3}$. It can be observed that for the lowest $N_{a}$ value, most of the current is concentrated at the bottom interface of the structure whereas it is homogenously spread all over the junction for $N_{a}=1 \times 10^{19} \mathrm{~cm}^{-3}$. Simulations have shown that this difference in the current distribution appears around $N_{a}=1 \times 10^{17} \mathrm{~cm}^{-3}$. Consequently, the active junction surface that really contributes to the dark current becomes wider when $N_{a}$ increases and it is expected that the ratio between the radius $R$ of the wire (related to the illuminated area) and the wirelength $L$ (related to the $p-n$ junction) will affect the photovoltaic performance of the wire solar cell as soon as high doping density in the core is reached. This is also illustrated in Figure 7 where the dark current characteristics are plotted for two wirelength values $L=25 \mu \mathrm{m}$ and $L=250 \mu \mathrm{m}$. For $N_{a}=1 \times 10^{17} \mathrm{~cm}^{-3}$, the high forward bias dark current does not depend on the wirelength 

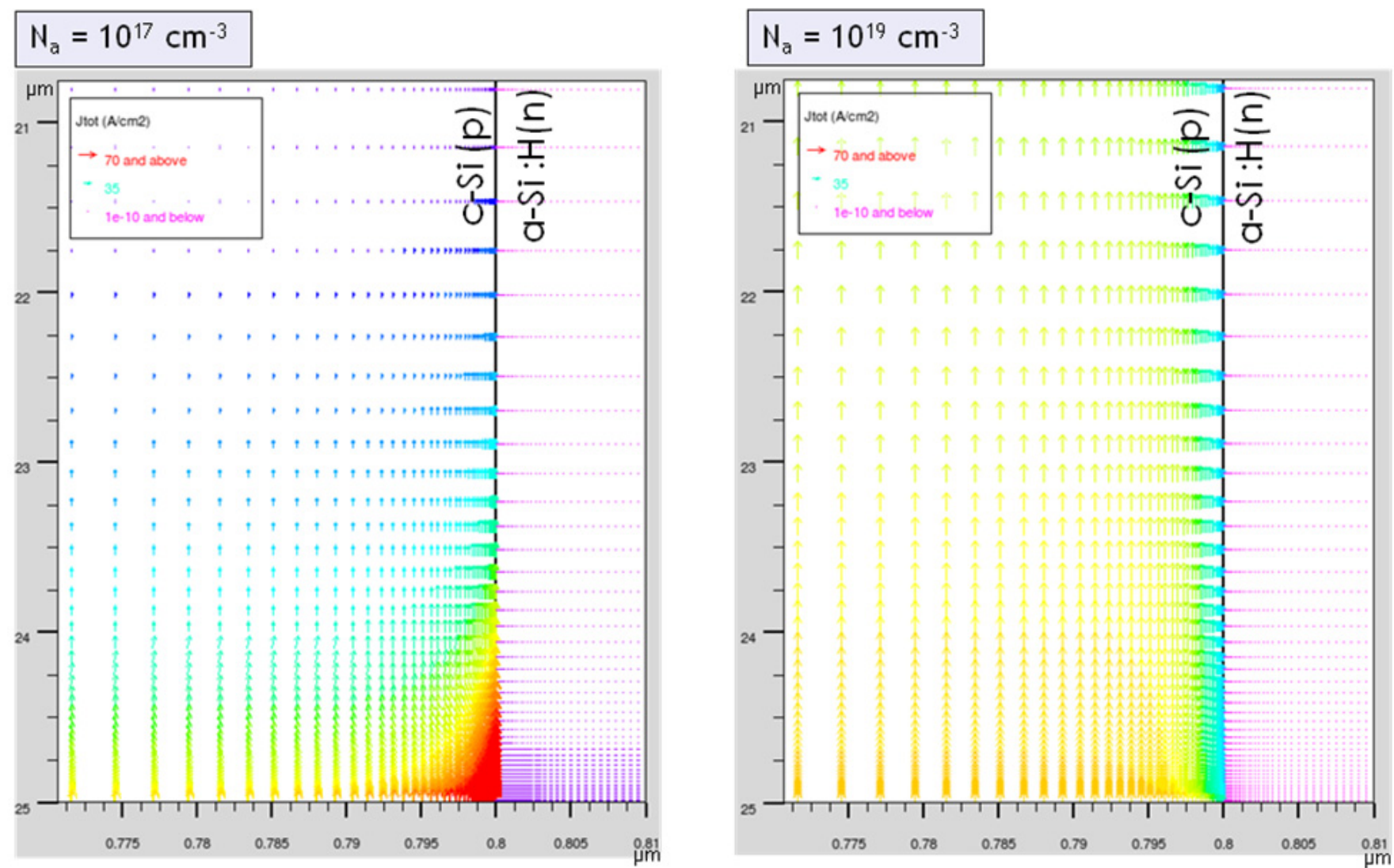

Fig. 6. Mapping of the dark current density distribution near the c-Si $(p) / \mathrm{a}-\mathrm{Si}: \mathrm{H}(n)$ heterointerface of the wire for $N_{a}=$ $1 \times 10^{17} \mathrm{~cm}^{-3}$ and $N_{a}=1 \times 10^{19} \mathrm{~cm}^{-3}$. Most of the current is concentrated at the bottom interface for the lowest value of $N_{a}$ whereas it is homogeneously spread all over the $p$ - $n$ junction for the highest one.

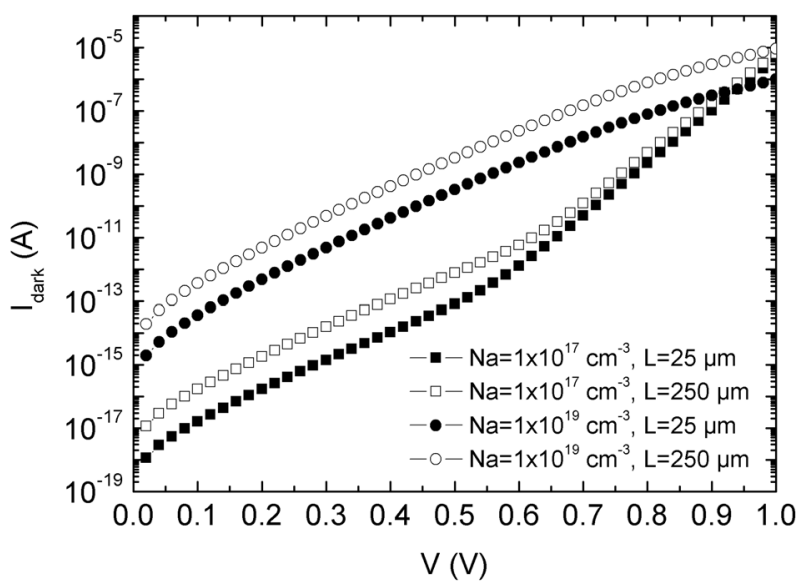

Fig. 7. Forward dark current characteristics $I_{\text {dark }}(V)$ for two wirelength values $(L=25 \mu \mathrm{m}$ and $L=250 \mu \mathrm{m})$ at $N_{a}=$ $1 \times 10^{17} \mathrm{~cm}^{-3}$ and $N_{a}=1 \times 10^{19} \mathrm{~cm}^{-3}$.

whereas it increases with $L$ for $N_{a}=1 \times 10^{19} \mathrm{~cm}^{-3}$ due to an homogeneous current distribution all over the wirelength. Thus, at high values of $N_{a}$, we should obtain a comparable decrease in $V_{o c}$ between a planar and a radial junction for an aspect ratio (ratio between the illuminated area and the junction area) of the wire equal to 1 . In the wire structures we have studied, the junction area is bigger than the illuminated area (ratio $R / L$ much smaller than 1) such that the drop in $V_{o c}$ with an increasing doping density is much more pronounced for the single-wire cell than for the planar one.

At low doping density, the current is dominated by the minority carriers diffusion and it mainly runs through a small active surface of the junction at the bottom of the structure as shown in Figure 6. This specific behavior seems to be related to the presence of a highly conductive electron interface layer at the $\mathrm{c}-\mathrm{Si}(p)$ surface. Indeed, the band offsets between a-Si:H and c-Si can provide a strong band bending which results in a strong inversion layer at the c-Si surface [11-13]. For c-Si $(p) / \mathrm{a}-\mathrm{Si}: \mathrm{H}(n)$ structures, it has been shown from numerical calculations that the inversion layer occurs even at low values of the conduction band offset $\Delta E_{c}$ and that the electron concentration in the interface region of c-Si strongly increases with $\Delta E_{c}$ as soon as $\Delta E_{c} \geqslant 0.1 \mathrm{eV}$ [12]. In the case of c-Si $(n) / \mathrm{a}-\mathrm{Si}: \mathrm{H}(p)$ structures, a lower limit of the valence band offset $\Delta E_{v}$ has been found equal to $0.25 \mathrm{eV}$ [13] such that $\Delta E_{v}$ can be varied in a wider range than $\Delta E_{c}$ to observe or not an interface inversion layer. Thus, in 

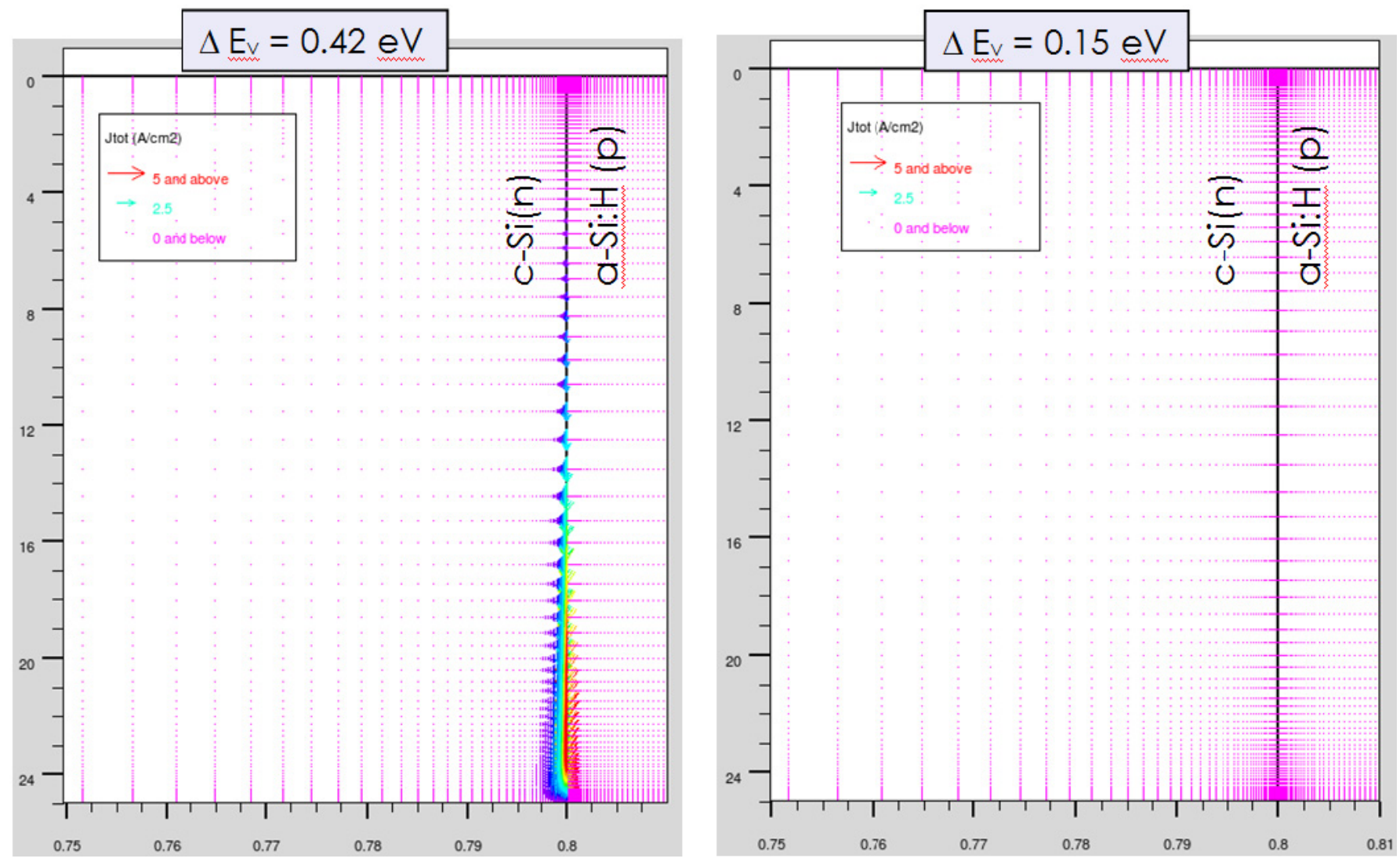

Fig. 8. Mapping of the dark current density distribution near the c-Si $(n) / \mathrm{a}-\mathrm{Si}: \mathrm{H}(p)$ heterointerface of the wire with $\Delta E_{v}=$ $0.42 \mathrm{eV}$ and $\Delta E_{v}=0.15 \mathrm{eV}$. For the highest value of $\Delta E_{v}$, most of the current is concentrated at the bottom interface in the crystalline core. For $\Delta E_{v}=0.15 \mathrm{eV}$, an homogeneous current distribution is obtained all along the heterointerface.

order to establish a link between the current distribution at the wire heterointerface and the presence-or not of an inversion surface layer, we have made some calculations on a c-Si $(n)$ / a-Si:H $(p)$ radial cell for different values of the valence band offset. A mapping of the dark current components near the c-Si $(n) / \mathrm{a}-\mathrm{Si}: \mathrm{H}(p)$ interface is given in Figure 8 for $\Delta E v=0.42 \mathrm{eV}$. and $\Delta E v=0.15 \mathrm{eV}$. It can be observed that the current distribution is homogeneous along the wire for $\Delta E v=0.15 \mathrm{eV}$ whereas it is concentrated at the bottom of the cell for $\Delta E v=0.42 \mathrm{eV}$ which can be explained by a charge accumulation in the inversion layer at the interface. Furthermore, calculations performed on a single wire crystalline homojunction also show an homogeneous current distribution all along the wire interface whatever the doping density in the $p$-type core as shown in Figure 9. The $I_{\text {dark }}(V)$ characteristics follow the exponential law given above with a dependence on the wirelength (not shown here). To come back to our c-Si $(p) / \mathrm{a}-\mathrm{Si}: \mathrm{H}(n)$ wire structures, high values of the doping density in the crystalline core lead to a modification of the band bending such that no strong inversion layer occurs. An homogeneous current distribution is thus observed along the wire for $N_{a} \geqslant 1 \times 10^{17} \mathrm{~cm}^{-3}$. Further studies have to be done to explain more precisely the location of the "hot spot" at the bottom of the interface in the case of a non homogeneous current distribution. In particular, the geometry and position of the contacts can play a important role in that feature.

\section{Conclusion}

We have studied the potential performance of a single c-Si $(p) / \mathrm{a}-\mathrm{Si}: \mathrm{H}(n)$ wire based solar cell through 2D numerical modelling. We have noticed in particular a drastic drop of the open-circuit voltage when the $p$-type doping density $N_{a}$ of the wire core increases beyond an optimum value equal to $1 \times 10^{17} \mathrm{~cm}^{-3}$. This loss in $V_{o c}$ is linked to an increase of the dark current with $N_{a}$, the transport in the forward bias region being dominated by recombination current in the a-Si:H layer at high values of $N_{a}$. Moreover, the decrease in $V_{o c}$ is less pronounced for an equivalent planar c-Si $(p) / \mathrm{a}-\mathrm{Si}: \mathrm{H}(n)$ heterojunction, the illuminated area of the planar and the radial modelled structures being the same. This difference can be explained by a dependence of the dark current in the wire with the wirelength. Thus, for $N_{a} \geqslant 1 \times 10^{17} \mathrm{~cm}^{-3}$ the wire based solar cell performance strongly depend on the ratio between the radius, which is linked to the illuminated area, and the wirelength, which is related to the $p-n$ junction area. In conclusion, this study shows that the doping density $N_{a}$ of the c-Si core should 

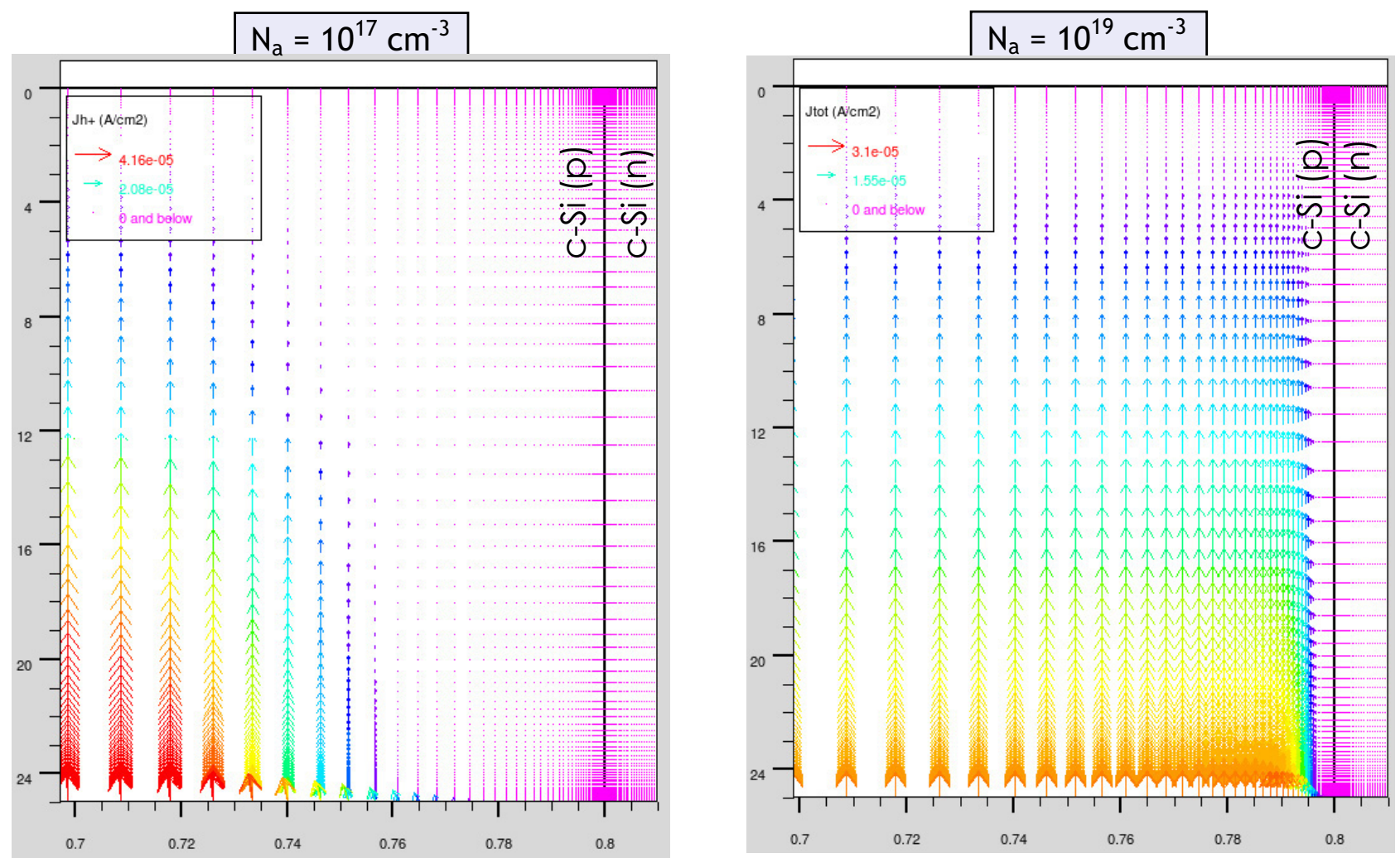

Fig. 9. Mapping of the dark current density distribution near the interface of a silicon crystalline homojunction wire with a doping density of the core $N_{a}=1 \times 10^{17} \mathrm{~cm}^{-3}$ and $N_{a}=1 \times 10^{19} \mathrm{~cm}^{-3}$.

be kept below an optimum value of $1 \times 10^{17} \mathrm{~cm}^{-3}$ and that any VLS catalyst which introduces a higher $p$-type doping density in the core should be avoided.

This work has been supported by French Research National Agency (ANR) through Habitat intelligent et solaire photovoltaïque program (Projet Siflex No. ANR-08-HABISOL-010).

\section{References}

1. M. Law, J. Goldberger, P. Yang, Annu. Rev. Mater. Res. 34, 83 (2004)

2. B.M. Kayes, H.A. Atwater, N.S. Lewis, J. Appl. Phys. 97, $114302(2005)$

3. L. Tsakalakos, J. Balch, J. Fronheiser, B.A. Korevaar, O. Sulima, J. Rand, Appl. Phys. Lett. 91, 233117 (2007)

4. B. Tian, X. Zheng, T.J. Kempa, Y. Fang, N. Yu, G. Yu, J. Huang, C.M. Lieber, Nature 449, 885 (2007)
5. T. Stelzner, M. Pietsch, G. Andrä, F. Falk, E. Ose, S. Christiansen, Nanotechnology 19, 295203 (2008)

6. M. Kelzenberg, S. Boettcher, J. Petykiewicz, D.B. TurnerEvans, M.C. Putman, E.L. Warren, J.M. Spurgeon, R.M. Briggs, N.S. Lewis, H.A. Atwater, Nat. Mater. 9, 239 (2010)

7. R.S. Wagner, C.W. Ellis, Appl. Phys. Lett. 4, 89 (1964)

8. D.E. Perea, J.E. Allen, S.J. May, B.W. Wessels, D.N. Seidman, L.J. Lauhon, Nano Lett. 6, 181 (2006)

9. V. Schmidt, J.V. Wittemann, U. Gösele, Chem. Rev. 110, 361 (2010)

10. ATLAS Users' Manual (Silvaco International, 2010)

11. J.P. Kleider, A.S. Gudovskikh, P. Roca i Cabarrocas, Appl. Phys. Lett. 92, 162101 (2008)

12. J.P. Kleider, Y.M. Soro, R. Chouffot, A.S. Gudovskikh, P. Roca i Cabarrocas, J. Damon-Lacoste, D. Eon, P.J. Ribeyron, J. Non-Cryst. Solids 354, 2641 (2008)

13. O.A. Maslova, J. Alvarez, E.V. Gushina, W. Favre, M.E. Gueunier-Farret, A.S. Gudovskikh, A.V. Ankudinov, E.I. Terukov, J.P. Kleider, Appl. Phys. Lett. 97, 252110 (2010) 